\title{
Kurt Gödel and Computability Theory
}

\author{
Richard Zach ${ }^{\star}$ \\ Department of Philosophy \\ University of Calgary \\ Calgary, AB T2N 1N4, Canada \\ rzach@ucalgary.ca
}

\begin{abstract}
Although Kurt Gödel does not figure prominently in the history of computabilty theory, he exerted a significant influence on some of the founders of the field, both through his published work and through personal interaction. In particular, Gödel's 1931 paper on incompleteness and the methods developed therein were important for the early development of recursive function theory and the lambda calculus at the hands of Church, Kleene, and Rosser. Church and his students studied Gödel 1931, and Gödel taught a seminar at Princeton in 1934. Seen in the historical context, Gödel was an important catalyst for the emergence of computability theory in the mid 1930s.
\end{abstract}

\section{Introduction}

Kurt Gödel's contributions to logic rank among the most important work in logic, and among the most important in 20th century mathematics. The theory of computability, and much of theoretical computer science more generally, has its roots, historically as well as conceptually, in the field of logic, and so it is a given that many of Gödel's results are also important in the field of theoretical computer science. However, it would be an exaggeration to say that Gödel was himself a pioneer of the field. That distinction belongs to those who lay the groundwork for a mathematical analysis of the concept of computation: Church, Kleene, Post, Rosser, and Turing, and those who followed in their footsteps. Nevertheless, the early work of Church, Kleene and Rosser was heavily influenced by Gödel, and it is perhaps not an exaggeration to say that their work was made possibly only by Gödel's earlier contributions.

The historical background both for Gödel's early work and that of Church, Rosser, and Kleene lies in the context of the foundational debate of the 1920s. Hilbert's program for the foundations of mathematics was the driving force behind many of the advances in logic during that time. His belief that all mathematical questions are in principle decidable underwrote his belief that the formal systems of mathematics considered then, such as arithmetic, analysis, and set theory, are complete in the sense that for any sentence $A$ in the respective language, either $A$ or $\neg A$ is derivable in the system. (Although Hilbert himself had

\footnotetext{
* Research supported by the Social Sciences and Humanities Research Council of
} Canada 
reservations whether this is the case in "higher domains", e.g., set theory, he did believe it was true for first and second-order arithmetic.) In a related sense, this was also the basis for Hilbert's conjecture that first-order logic is complete in the sense that any valid sentence is derivable from the axioms of the predicate calculus. (It was known by the mid 1920s that first-order logic is not complete in the first, syntactic sense described above - there are formulas $A$ such that neither $A$ nor $\neg A$ is derivable in first-order logic alone.) It was also the basis for his aim in the work on the decision problem for logic, i.e., that it should be possible to find a procedure to decide, for any given sentence of first-order logic, whether it is provable from the axioms of the predicate calculus or not. Hilbert's firm belief that classical mathematics is secure in the sense that the axioms of arithmetic and set theory do no lead to contradictions suggested that it should be possible to prove that these axioms are consistent, and since the statement of consistency is a purely combinatorial one about what sequences of formulas of certain sorts there are, that consistency could be proved using elementary, "finitary" methods. These methodologically motivated questions, then, guided the work of the Hilbert school: to solve the decision problem by giving a decision problem for predicate logic; to prove that arithmetic and logic are complete; and to find a finitary consistency proof of arithmetic and analysis.

In 1929 and 1930, Gödel solved the latter two problems. In his dissertation (1929; 1930), he showed that first-order logic is complete, and in his Habilitationsschrift $(1930 ; 1931)$ he showed that arithmetic is incomplete. Very soon afterward he himself accepted the consequence of the second incompleteness theorem that no finitary consistency proof of arithmetic can be given, a consequence that others (e.g., von Neumann and Herbrand) accepted more readily. Although Church and Turing gave the definitive (negative) solution to the decision problem, Gödel also actively contributed to the literature on Hilbert's first task (Gödel, 1932, 1933).

Church's first publications on the $\lambda$-calculus were similarly concerned with foundational problems in mathematics: Church's stated aim was to develop a new axiomatization of logic which avoids the paradoxes, but in a manner different from Russell's theory of types or axiomatic set theory. Although we now think of the (simple) $\lambda$-calculus as a formalism for expressing computable functions, Church did not originally conceive of it in that way - for him, the system which evolved into the $\lambda$-calculus was a logical formalism which, he hoped, would be capable of serving as a contradiction-free formalization of mathematics. Unfortunately, Church's original system proved to be inconsistent (Kleene and Rosser, 1935). Kleene's and Rosser's proof that it was inconsistent made essential use of the method of Gödel coding introduced in (Gödel, 1931). Kleene's (1935) development of arithmetic and the representability of recursive functions within the $\lambda$-calculus was motivated, in part, by the aim of reproducing Gödel's incompleteness result in the context of the $\lambda$-calculus, and his important normal form theorem also relied on Gödel coding. It was in the context of this turn towards metamathematical investigations of the $\lambda$-calculus along the lines of Gödel (1931) that the notion of $\lambda$-definability achieved pride of place in the work of Church, 
Kleene, and Rosser. The positive results obtained by Kleene to the effect that a great many recursive functions could be formalized in the $\lambda$-calculus led Church to formulate what now has come to be known as Church's Thesis, viz., that every effectively computable function is $\lambda$-definable. And again it was Gödel, who at the time (1934) was in Princeton, who led Church and his students to take a broader view: his skepticism about Church's thesis when first formulated regarding $\lambda$-definability and his proposal that general recursiveness might be a better candidate for a precise characterization of effective computability led Kleene to show that the two notions are coextensive: every $\lambda$-definable function is general recursive and conversely (Kleene, 1936b).

In what follows, I will give an outline of the early history of recursion theory, with special emphasis on the role Gödel and his results played in it. In my survey of these developments, I rely heavily on the recollections of Kleene (1981; 1987) and the analyses of Davis (1982) and Sieg (1997), as well as chapter V of Dawson's (1997) biography of Gödel.

\section{Church's System and Gödel's Incompleteness Result}

In the years 1929-1931, Church developed an alternative formulation of logic (Church, 1932, 1933), which he hoped would serve as a new foundation of mathematics which would avoid the paradoxes. Church taught a course on logic in the Fall of 1931, where Kleene, then a graduate student, took notes. During that time, Church and Kleene were first introduced to Gödel's work on incompleteness: the occasion was a talk by John von Neumann on Gödel's work. Church and Kleene immediately studied the paper in detail. At the time, it was not yet clear how general Gödel's results were. Church believed that the incompleteness of Gödel's system $P$ (a type-theoretic higher-order formulation of Peano arithmetic) relies essentially on some feature of type theory, and that Gödel's result would not apply to Church's own system. It nevertheless seems like it became a pressing issue for Church to determine to what extent Gödel's results and methods could be carried out in his system. He set Kleene to work on the task of obtaining Peano arithmetic in the system. Kleene succeeded in carrying this out in the first half of 1932. It involved, in particular, showing that various numbertheoretic functions are $\lambda$-definable. In July 1932, Gödel wrote to Church, asking if Church's system could be proved consistent relative to Principia Mathematica. Church was skeptical of the usefulness of such a relative consistency proof. He wrote,

In fact, the only evidence for the freedom from contradiction of Principia Mathematica is the empirical evidence arising from the fact that the system has been in use for some time, many of its consequences have been drawn, and no one has found a contradiction. If my system be really free from contradiction, then an equal amount of work in deriving its consequences should provide an equal weight of empirical evidence for its freedom from contradiction. [...] 
But it remains barely possible that a proof of freedom from contradiction for my system can be found somewhat along the lines suggested by Hilbert. I have, in fact, made several unsuccessful attempts to do this.

Dr. von Neumann called my attention last fall to your paper entitled "Über formal unentscheidbare sätze der Principia Mathematica." I have been unable to see, however, that your conclusions in $\S 4$ of this paper apply to my system. Possibly your argument can be modified so as to make it apply to my system, but I have not been able to find such a modification of your argument. (Church to Gödel, July 27, 1932. Gödel 2003a, 368-369)

Section $\S 4$ of Gödel (1931) which Church mentions here is the section in which Gödel sketched the second incompleteness theorem. Since Gödel did not provide a complete proof of the theorem - indeed, the first complete proof did not appear until Hilbert and Bernays (1939) — Church was surely justified in doubting that the result applies to his system. It leaves open the question, however, of whether Church believed, at the time, that the construction of the first incompleteness theorem do go through in his system.

Kleene reports (Crossley, 1975) that he carried out the development of Peano arithmetic in Church's system between January and June 1932, and then wrote up the results over the following year. The paper reporting these results (Kleene, 1935) was received by the American Journal of Mathematics on October 9, 1933, and in revised version on June 18, 1934. The paper also contains the arithmetization of syntax, making use of Gödel's methods and results, and a proof that all primitive recursive functions are $\lambda$-definable. Kleene also showed that for any formula in the formalism of Principia Mathematica, the question of whether it is provable is equivalent to the question of whether a certain expression of Church's system has a normal form. Only a few months after Kleene submitted the final version of his paper, in November 1934, Rosser and he submitted another paper to the Annals of mathematics (Kleene and Rosser, 1935). In it, they showed that Church's system, as well as Curry's combinatory logic (Curry, 1930), were inconsistent. In their proof, they again made extensive use of Gödel's arithmetization of syntax, and were able to derive a version of Richard's paradox within the system. The fragment of Church's system with the logical axioms removed is demonstrably consistent: it is the simple $\lambda$-calculus (see Barendregt 1997 for the impact of $\lambda$-calculus in computer science, and Seldin 2006 for a history of the $\lambda$-calculus).

Church, then, turned out to be right: Gödel's second incompleteness theorem does not apply to his system-because the theorem only applies to consistent formal systems. But in order to obtain this result, and many of the positive results due to Kleene which provided the foundation for Church's undecidability results a year later, Gödel's methods were of crucial importance, both because they motivated a certain line of inquiry and because Kleene, Rosser, and Church were able to build on them.

The methods introduced in Gödel (1931) and used by Kleene and Rosser to show that Church's system was inconsistent also figure prominently in Church's 
negative solution of the decision problem. Church $(1935,1936 \mathrm{~b})$ first showed that the question of whether a given expression of the $\lambda$-calculus has a normal form is not recursive. In the same paper, Church also stated what is now known as "Church's Thesis," viz., that the general recursive functions (and hence, the $\lambda$-definable ones) are just the "effectively computable" ones. The theorem and the thesis combine to yield the result that having a normal form is not an effectively decidable property. The genesis of Church's Thesis will be outlined in the next section. Here, I want to stress only that the result itself, and with it the negative solution of the decision problem for first-order logic (Church, 1936a), made essential use of Gödel's work.

Kleene (1987) himself emphasizes the importance of Gödel (1931) in the work that he and Rosser carried out in their seminal contributions to recursion theory in the early 1930s:

After the colloquium [by von Neumann in the fall of 1931], Church's course continued uninterruptedly concentrating on his formal system; but on the side we all read Gödel's paper, which to me opened up a whole new world of fascinating ideas and perspectives.

\section{Gödel and Church's Thesis}

Gödel's (1931) had a dramatic and lasting influence on the pioneers of recursion theory and the development of the $\lambda$-calculus. Gödel had a more direct and personal influence in the formation of Church's thesis. He visited Princeton in the 1933/34 academic year and gave a series of lectures there between February and May 1934, which was attended by Church, Kleene, and Rosser. Kleene's work on defining various number-theoretic functions in the $\lambda$-calculus (1935) first prompted Church to put forward a tentative version of the thesis in late 1933 or early 1934, in the form: every effectively calculable function is $\lambda$-definable. In conversation, Gödel expressed skepticism about the thesis.

Towards the end of his Princeton lectures, Gödel introduced the notion of general recursive function. This notion was based on a suggestion by Herbrand in a letter to Gödel of April 7, 1931 (Gödel, 2003b, 14-21). In the lectures, Gödel (1934, 368-369), defined the general recursive functions as those which can be computed using a specific set of substitution rules from a set of defining equations, and for which the result of the computation is uniquely determined. (For a discussion of the connection between Herbrand's and Gödel's notions, see Sieg 2005.) Gödel did not at first propose the definition of general recursive function as an explication of the informal notion of "effectively computable," but only as an explication of the notion of "recursive function." In 1931, Gödel had introduced the primitive recursive functions (although he called them then just "recursive functions"). It was already known since the mid-1920s (Hilbert, 1926; Ackermann, 1928) that there are non-primitive recursive functions which can be defined by double recursion, and in the early 1930s, Péter $(1934,1935)$ studied such recursive functions in more detail. 
Gödel was interested in a precise characterization of intuitively recursive functions. Kleene (1936b) soon succeeded in establishing that the general recursive functions are exactly the $\lambda$-definable ones, and this seems to have been a reason for Church to propose his thesis in print in 1935. Kleene (1936a) is a systematic study of Gödel's class of general recursive functions. It contains Kleene's normal form theorem, that every general recursive function can be written as $f(\mu x[g(x)=0])$, with $f, g$ primitive recursive, Kleene's $T$ predicate, and examples of non-recursive functions and relations based on it.

For a more detailed historical discussion on the origin of Church's Thesis and Gödel's influence, see Davis (1982) and Sieg (1997).

\section{Gödel and Complexity Theory}

Another work of Gödel's played a role in the development and gradual acceptance of Church's Thesis - although Gödel himself apparently became convinced of the truth of the thesis only through Turing's work. That work was an abstract on length of proofs (Gödel, 1936). In stating his Thesis, Church (1936b, §7) had introduced the notion of functions computable in a logic $S: f$ is is computable in $S$ if there is some term $\phi$ so that for every numeral $m$ there is a numeral $n$ with $S \vdash \phi(m)=n$ iff $f(m)=n$ (following Kleene 1952, §59, such functions are also called reckonable in $S$ ). In a note added in proof, Gödel (1936) remarked that this notion of computability is absolute, in the sense that if a function is computable in a higher-order system $S$, it already is computable in first-order arithmetic - i.e., the general recursive functions are all the functions computable in any consistent system $S$ containing arithmetic. The reason for this is, of course, that if the system is formal in the sense that its proofs are recursively enumerable, then then function is computable by searching through all proofs until one finds one of $\phi(m)=n$, and this procedure is insensitive to the logical strength of the theory $S$. This result served both Church and later also Gödel as evidence for the Church-Turing Thesis (see Gödel 1946 and Sieg 1997, 2006).

The main part of (Gödel, 1936), however, was not concerned with computability so much as with proof complexity. The result that Gödel announced concerned speed-up of proofs (measured as number of symbols) between $n$-th and $(n+1)$ storder arithmetic. (Buss 1994 contains a proof of the result.) 20 years later, Gödel was again thinking about proof complexity. In an intriguing letter to John von Neumann on March 20, 1956 (Gödel, 2003b, 372-377), Gödel discussed the complexity of deciding for a formula $A$ of first-order logic, whether $A$ has a proof with $k$ symbols or less. Cook has shown that this problem is NP-complete (see Hartmanis 1989 and Buss 1995).

Unlike Gödel's earliest work, his thoughts on proof complexity and feasible computation in the letter to von Neumann had no impact on the historical development of computability and complexity theory. It nevertheless shows that questions of the nature of computability, even though they were not at the forefront of Gödel's thought or prominent in his publications, did occupy Gödel throughout his professional career. 


\section{Bibliography}

Ackermann, Wilhelm. 1928. Zum Hilbertschen Aufbau der reellen Zahlen. Mathematische Annalen 99: 118-133.

Barendregt, Henk. 1997. The impact of the lambda calculus in logic and computer science. The Bulletin of Symbolic Logic 3(2): 181-215.

Buss, Samuel R. 1994. On Gödel's theorems on lengths of proofs I: Number of lines and speedups for arithmetic. Journal of Symbolic Logic 39: 737-756.

Buss, Samuel R. 1995. On Gödel's theorems on lengths of proofs II: Lower bounds for recognizing $k$ symbol provability. In Feasible Mathematics II, eds. P. Clote and J. Remmel, 57-90. Basel: Birkhäuser.

Church, Alonzo. 1932. A set of postulates for the foundation of logic. Annals of Mathematics 33: 346-366.

Church, Alonzo. 1933. A set of postulates for the foundation of logic (second paper). Annals of Mathematics 34: 839-864.

Church, Alonzo. 1935. An unsolvable problem of elementary number theory. Bulletin of the American Mathematical Society 41: 332-333.

Church, Alonzo. 1936a. A note on the Entscheidungsproblem. Journal of Symbolic Logic 1: 40-41.

Church, Alonzo. 1936b. An unsolvable problem of elementary number theory. American Journal of Mathematics 58: 345-363.

Crossley, J. N. 1975. Remniscences of logicians. In Algebra and Logic. Papers from the 1974 Summer Research Institute of the Australian Mathematical Society, ed. J. N. Crossley, LNM 450, 1-62. Berlin: Springer.

Curry, Haskell B. 1930. Grundlagen der kombinatorischen Logik. American Journal of Mathematics 52: 509-536, 789-834.

Davis, Martin. 1982. Why Gödel didn't have Church's thesis. Information and Control 54: 3-24.

John W. Dawson, Jr. 1997. Logical Dilemmas. The Life and Work of Kurt Gödel. Wellesley, Mass.: A K Peters.

Gödel, Kurt. 1929. Über die Vollständigkeit des Logikkalküls. Dissertation, Universität Wien. Reprinted and translated in (Gödel, 1986, 60-101).

Gödel, Kurt. 1930. Die Vollständigkeit der Axiome des logischen Funktionenkalküls. Monatshefte für Mathematik und Physik 37: 349-360. Reprinted and translated in (Gödel, 1986, 102-123).

Gödel, Kurt. 1930. Einige metamathematische Resultate über Entscheidungsdefinitheit und Widerspruchsfreiheit. Anzeiger der Akademie der Wissenschaften in Wien 67: 214-215. Reprinted and translated in (Gödel, 1986, 140-143).

Gödel, Kurt. 1931. Über formal unentscheidbare Sätze der Principia Mathematica und verwandter Systeme I. Monatshefte für Mathematik und Physik 38: 173-198. Reprinted and translated in (Gödel, 1986, 144-195).

Gödel, Kurt. 1932. Ein Spezialfall des Entscheidungsproblem der theoretischen Logik. Ergebnisse eines mathematischen Kolloquiums 2: 27-28. Reprinted and translated in (Gödel, 1986, 130-235). 
Gödel, Kurt. 1933. Zum Entscheidungsproblem des logischen Funktionenkalküls. Monatshefte für Mathematik und Physik 40: 433-443. Reprinted and translated in (Gödel, 1986, 306-327).

Gödel, Kurt. 1934. On undecidable propositions of formal mathematical systems. Lecture notes by Stephen C. Kleene and J. Barkely Rosser, Princeton University. Reprinted in (Gödel, 1986, 338-371).

Gödel, Kurt. 1936. Über die Länge von Beweisen. Ergebnisse eines mathematisches Kolloquiums 7: 23-24. Reprinted and translated in (Gödel, 1986, 394-399).

Gödel, Kurt. 1946. Remarks before the Princeton bicentennial conference on problems in mathematics. In Collected Works, eds. Solomon Feferman et al., vol. 2, 144-153. Oxford: Oxford University Press.

Gödel, Kurt. 1986. Collected Works, vol. 1, eds. Solomon Feferman et al. Oxford: Oxford University Press.

Gödel, Kurt. 2003a. Collected Works, vol. 4, eds. Solomon Feferman et al. Oxford: Oxford University Press.

Gödel, Kurt. 2003b. Collected Works, vol. 5, eds. Solomon Feferman et al. Oxford: Oxford University Press.

Hartmanis, Juris. 1989. Gödel, von Neumann and the $\mathrm{P}=$ ?NP problem. Bulletin of the European Association for Theoretical Computer Science (EATCS) 38: 101-107.

Hilbert, David. 1926. Über das Unendliche. Mathematische Annalen 95: 161-90. Lecture given Münster, 4 June 1925. English translation in (van Heijenoort, 1967, 367-392).

Hilbert, David and Paul Bernays. 1939. Grundlagen der Mathematik, vol. 2. Berlin: Springer.

Kleene, Stephen C. 1935. A theory of positive integers in formal logic. American Journal of Mathematics 57: 153-173, 219-244.

Kleene, Stephen C. 1936a. General recursive functions of natural numbers. Mathematische Annalen 112: 727-742.

Kleene, Stephen C. 1936b. $\lambda$-definability and recursiveness. Duke Mathematical Journal 2: 340-353.

Kleene, Stephen C. 1952. Introduction to Metamathematics. Amsterdam: NorthHolland.

Kleene, Stephen C. 1981. Origins of recursive function theory. Annals of the History of Computing 3: 52-67.

Kleene, Stephen C. 1987. Gödel's impression on students of logic in the 1931s. In Gödel Remembered, eds. Paul Weingartner and Leopold Schmetterer, 49-64. Bibliopolis.

Kleene, Stephen C. and J. Barkley Rosser. 1935. The inconsistency of certain formal logics. Annals of Mathematics 36: 630-636.

Péter, Rósza. 1934. über den Zusammenhang der verschiedenen Begriffe der rekursiven Funktionen. Mathematische Annalen 110: 612-632.

Péter, Rósza. 1935. Konstruktion nichtrekursiver Funktionen. Mathematische Annalen 111: 42-60. 
Seldin, Jonathan P. 2006. The logic of Curry and Church. In Handbook of the History of Logic, eds. Dov Gabbay and John Woods, vol. 5. Amsterdam: Elsevier. Forthcoming.

Sieg, Wilfried. 1997. Step by recursive step: Church's analysis of effective computability. Bulletin of Symbolic Logic 3: 154-180.

Sieg, Wilfried. 2005. Only two letters: The correspondence between Herbrand and Gödel. Bulletin of Symbolic Logic 11: 172-184.

Sieg, Wilfried. 2006. Gödel on computability. Philosophia Mathematica 14. Forthcoming.

van Heijenoort, Jean, ed. 1967. From Frege to Gödel. A Source Book in Mathematical Logic, 1897-1931. Cambridge, Mass.: Harvard University Press. 